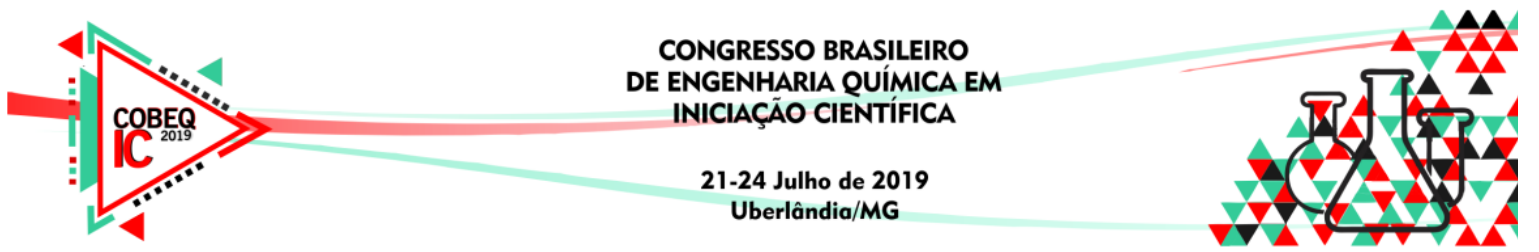

\title{
Otimização experimental e voltamétrica para a identificação e quantificação do desregulador endócrino Bisfenol A
}

\author{
L. M. ANDRINO ${ }^{1}$ e D. De SOUZA ${ }^{1}$ \\ ${ }^{1}$ Universidade Federal de Uberlândia, Campus Patos de Minas \\ E-mail para contato: laviniandrino@ hotmail.com
}

\begin{abstract}
RESUMO - O objetivo deste trabalho foi o desenvolvimento de metodologia analítica para a identificação e quantificação do desregulador endócrino Bisfenol A (BPA) e posterior aplicação em amostras de alimentos industrializados e armazenados em embalagens plásticas. Para isto, foram empregados métodos eletroanalíticos, nos quais as intensidades de sinais de correntes elétricas são empregadas para avaliação das concentrações de BPA e para avaliação da contaminação em alimentos industrializados armazenados em embalagens plásticas. O eletrodo sólido de amalgama de prata, será o sensor voltamétrico empregado, pois apresenta características semelhantes às do eletrodo de mercúrio, porém sem geração de resíduos tóxicos e com excelente reprodutibilidade nos sinais analíticos. A técnica analítica que será utilizada será a voltametria de onda quadrada, por ser, juntamente com o eletrodo sólido de amálgama de prata, uma junção que apresenta alta sensibilidade, seletividade, repetibilidade e reprodutibilidade. Os parâmetros experimentais (eletrólito de suporte e pH do meio) serão avaliados para obtenção de maior sensibilidade e seletividade. Além disto, os parâmetros da técnica de voltametria de onda quadrada também serão otimizados para obtenção de adequada sensibilidade e seletividade, além de estudos sobre mecanismo de reações químicas de BPA em diferentes tipos de amostras. Assim poderá ser possível avaliar se as embalagens estudadas apresentam limites aceitáveis para a presença de BPA, de acordo com o limite exigido pela legislação, e se ocorre contaminação dos alimentos por tempo de armazenamento e aquecimento.
\end{abstract}

\section{INTRODUÇÃO}

O Bisfenol A (BPA, ou 4,4-di-hidróxi-2,2-difenilpropano) é usado como monômero em vários polímeros para fabricação de resinas époxi e policarbonatos, os quais podem ser empregados na fabricação de embalagens de bebidas e de alimentos. Assim, são usados em larga escala nas indústrias, pois apresenta baixo custo de produção e possui uma alta versatilidade e propriedades que conferem uma maior resistência, flexibilidade e estabilidade aos materiais. Deste modo, também é encontrado em diversas embalagens como, mamadeiras, embalagens retornáveis de água, cerveja e refrigerante. Além disso, está presente no revestimento de latas de conservas e frascos de alimentos para bebês. O BPA é um composto que possui elevada toxidade, afetando a saúde humana de forma direta e indireta. Na forma, direta ocorre a contaminação pela ingestão de alimento contaminado, por exemplo o peixe que é contaminado por águas que contém o BPA, depositado por resquícios durante as fabricações 


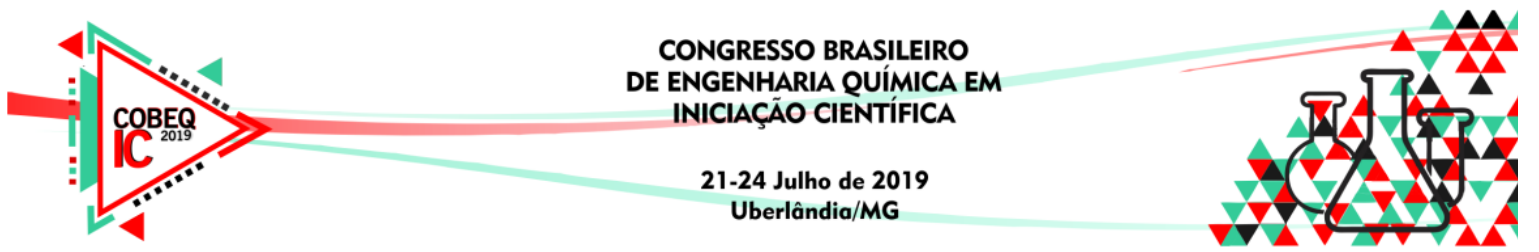

nas indústrias. Enquanto na forma indireta, ocorre a contaminação através da transferência do BPA da embalagem para o alimento, quando ocorre uma alteração na temperatura ou no tempo de armazenamento (SENCI, (2015)). A alta toxidade do BPA, provoca riscos à saúde humana mesmo em doses baixas, causando efeitos adversos, como diabetes, câncer, obesidade, puberdade precoce, neurotoxicidade, doenças cardiovasculares, problemas no desenvolvimento e é um desregulador endócrino (RAGAVAN et al, (2013)).

Assim é evidente, a necessidade do desenvolvimento de metodologias para identificar com resultados satisfatórios, de forma simples, sensível, seletiva e confiável a determinação do BPA. Os métodos analíticos para a detecção do BPA são diversos, porém assim como os métodos cromatográficos eles possuem dificuldades para a sua execução como, a necessidade de um pré-tratamento das amostras, o preço elevado da instrumentação e materiais empregados, a necessidade de mão de obra altamente capacitada para a realização dos experimentos e o maior tempo necessário para a realização da análise química. Desse modo, foram desenvolvidos métodos eletroanalíticos para a análise que utilizam as medidas de propriedades elétricas das soluções nos quais existem dois principais tipos de medidas: galvanostáticas (medidas de potencial elétrico) e potenciostáticas (medidas de correntes elétricas) (WANG, (2006)).

O sucesso da eletroanálise é relacionado à escolha adequada do sensor voltamétrico, pois é nele que ocorre a reação redox de interesse (oxidação e/ou redução), o qual deverá ser escolhido, observando algumas características como custo de fabricação, propriedades mecânicas, toxicidade e disponibilidade do material, relacionando-o com o analito que será usado (WANG, (2006)). Sendo assim, o interesse nos eletrodos sólidos de amálgamas metálicas tem aumentado, já que é composto por uma liga, contendo mercúrio e um ou mais metais, mas possui propriedades eletroquímicas semelhantes às do mercúrio, porém não é tóxico, sendo uma das suas principais vantagens a estabilidade mecânica. Por isso, o objetivo deste trabalho foi o desenvolvimento da metodologia eletroanalíticas para determinação de BPA empregando-se o eletrodo sólido de amálgama de prata (m-AgSAE), que apresenta uma facilidade na construção, rápida renovação da superfície e uma excelente reprodutibilidade (DE SOUZA et al, (2011)).

Para a verificação da aplicabilidade da metodologia desenvolvida, as amostras alimentícias serão avaliadas por meio da aplicação analítica realizada diretamente no alimento. As embalagens poliméricas, garantem a qualidade do produto durante o seu transporte e armazenamento. Porém, condições de armazenamento adversas podem gerar uma difusão de compostos da matriz polimérica para a matriz alimentícia, assim a embalagem deixa de exercer sua principal função de segurança para os alimentos e se torna um meio de transporte e difusão de substâncias tóxicas (STOFFERS, (2004)). Desta forma, serão analisadas as amostras observando os efeitos da potência e do tempo no micro-ondas, pois é por meio da radiação, em que ocorrer a transferência do BPA, após o aquecimento. Além disto, também serão avaliados alimentos armazenados e comercializados em embalagens plásticas, tais como água mineral, sucos e refrescos industrializados. De acordo com a Agência Nacional da Vigilância Sanitária (ANVISA), na resolução em que se refere as embalagens alimentícias, é definido um limite de migração específica (LME), para os principais constituintes nas embalagens plásticas que possuem teor tóxico. Nessa lista, um dos principais aditivos contidos é o BPA, um antioxidante que possui como LME (mg kg-1) o valor máximo de $0,6 \mathrm{mg} \mathrm{kg}^{-1} .5$ Dessa maneira, serão avaliados analiticamente várias amostras de alimentos e os resultados serão comparados para verificar também a qualidade de cada embalagem. 


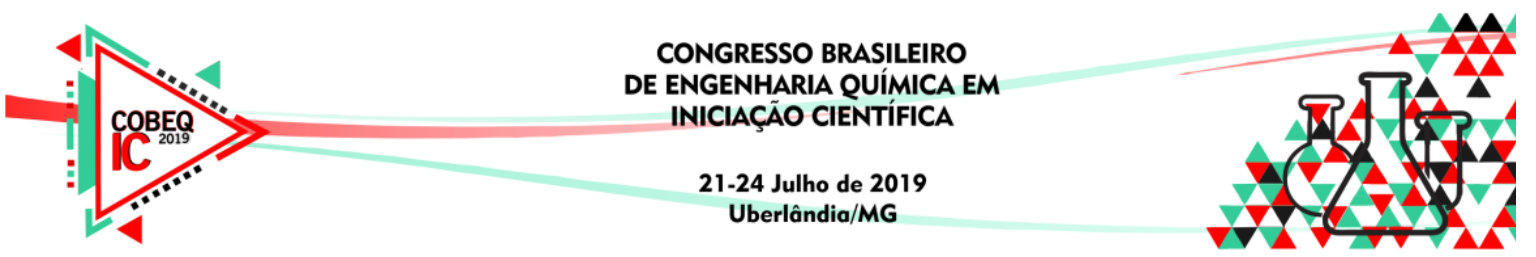

\section{EXPERIMENTAL}

A relação entre os parâmetros químicos e as medidas de corrente e potencial elétrico foram empregadas para identificação e quantificação do BPA. Para o uso de técnicas eletroanalíticas empregou-se uma célula eletroquímica, composta por um recipiente de vidro contendo três eletrodos (trabalho, referência e auxiliar), onde o eletrodo de trabalho foi o eletrodo sólido de amálgama de prata $(\mathrm{AgSAE})$ que é onde ocorreu a reação redox de interesse (redução do BPA), o eletrodo de referência $\left(\mathrm{Ag} / \mathrm{AgCl} 3,0 \mathrm{~mol} \mathrm{~L}^{-1}\right.$ de $\mathrm{KCl}$ ) que fornece um potencial estável e reprodutível e com quem o potencial do eletrodo de trabalho é comparado, e o eletrodo auxiliar (fio de platina) que que auxilia no transporte da corrente (DE SOUZA et $a l,(2004))$.

Todas as soluções foram preparadas utilizando a massa adequada ou o volume adequado de cada um dos reagentes analíticos de alta pureza, as quais foram: solução tampão Clark e Lubs, Tris cloridrato, Tetraborato, tampão fosfato, tampão Brinton-Robinson (BR), hidróxido de sódio e tampão fosfato-salino (PBS). Para o preparo das soluções foram utilizados, vidrarias comuns que foram lavadas com água da torneira, sabão, álcool e por último enxaguadas com água purificadas com um Sistema Purificador de água Osmose Reversa OS50 LX. Além disso, utilizou-se uma balança analítica classe I (Bel Engineering, M214A) com precisão de 0,0001g e o pHmêtro de bancada Mpa-210 (MS TECNOPON Instrumentação). Na realização dos experimentos o equipamento utilizado foi um Potenciostato/Galvanostato microAutolab III Metrohn-Pensalab, sendo controlado pelo software NOVA® 2.1, também da MetrohnPensalab.

Neste trabalho empregou-se a voltametria de onda quadrada (VOQ), onde a variação de potencial elétrico é realizada na forma de uma escada e as medidas da corrente são realizadas antes e depois de aplicar o pulso de potencial. Foi empregada esta técnica por ser uma das técnicas de pulso mais rápidas e sensíveis (DE SOUZA, (2004)). Antes de iniciar os experimentos de SWV, o AgSAE é polido em uma lixa de granulação 1500, para retirar qualquer resíduo. Após isto é lavado junto com os demais eletrodos e a célula eletroquímica e,pregando-se água e sabão neutro, seguidos de enxague com etanol e por fim água purificada. Primeiramente, coloca-se na célula uma solução $0,2 \mathrm{~mol} \mathrm{~L}^{-1} \mathrm{de} \mathrm{KCl}$, juntamente com os eletrodos e realiza-se o condicionamento eletroquímico do AgSAE aplicando-se -2,2 V por 600 segundos, seguidos por experimentos de Voltametria Ciclica com o eletrólito de suporte, para ocorrer uma adaptação da superfície a soluções muito distintas. Após a Voltametria Cíclica no eletrólito de suporte, inicia-se a SWV aplicando um potencial de -2,20 V em um tempo de $30 \mathrm{~s}$ entre cada experimento, promovendo-se assim completa renovação da superfície do AgSAE. Antes de iniciar as adições do Bisfenol A, sempre foi feito o branco, em que fez a SWV para verificar se o eletrodo estava limpo e sem contaminação.

\section{RESULTADOS E DISCUSSÃO}

Após a construção e ativação do AgSAE, iniciou-se os experimentos para otimização experimental onde foram avaliados alguns eletrólitos de suporte observando-se em que meio era possível obter maior intensidade de pico de redução (aumentando-se a sensibilidade) e com pico mais estreito (melhorando a seletividade) e também com o potencial de redução mais próximo de zero (mais favorável). Assim avaliou-se o eletrólito de suporte que melhor se 


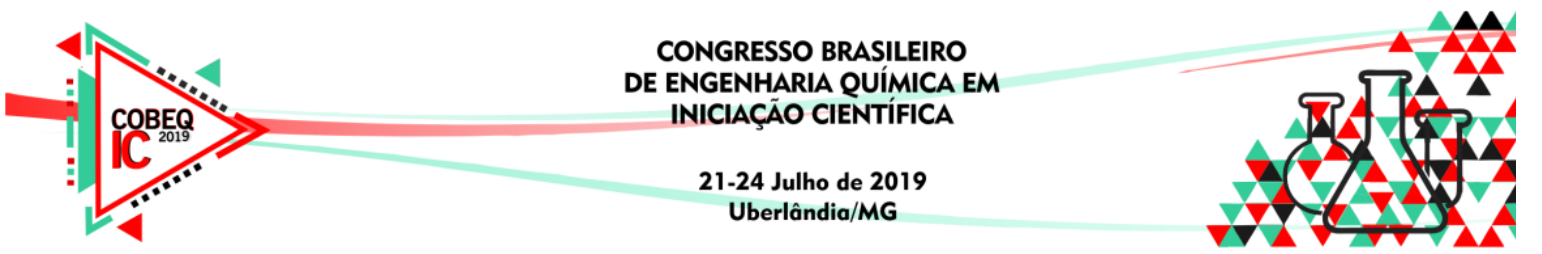

identificou aos requisitos, considerando-se três soluções com valores similares de $\mathrm{pH}$, onde-se observou-se que entre as soluções de tampão amônio, BR e PBS, todos com pH =9,0, a melhor resposta voltamétrica foi obtida em meio de tampão fosfato-salino (PBS), como pode ser visualizado na Figura 1.

Nas três soluções tampão, o Bisfenol A apresentou pico de redução bem definido, entretanto em meio de tampão PBS, o pico voltamétrico apresentou maior linearidade com o aumento de concentração e menor valor de potencial de redução, sendo mais adequado para o desenvolvimento da metodologia. Considerando-se o eletrólito tampão amônio, observa-se que não houve crescimento linear do sinal com a concentração e pico voltamétrico apresentou valor mais negativo que o do tampão PBS. Já em meio de solução tampão BR ocorreu um crescimento intensidade de corrente acompanhado por intenso deslocamento e alargamento do pico voltamétrico, o que não ocorreu no tampão PBS. Assim, variou-se $\mathrm{p} \mathrm{pH}$ da solução tampão PBS, onde observou-se que quanto menor o valor de $\mathrm{pH}$, maior a intensidade de sinal voltamétrico obtido, como está mostrado na Figura 2.

Figura 1: Voltamogramas de onda quadrada para a redução de diferentes concentrações de Bisfenol A sobre AgSAE em diferentes eletrólitos de suporte, $f=100 \mathrm{~s}^{-1}, \Delta \mathrm{E}_{\mathrm{s}}=-2 \mathrm{mV} \mathrm{e}$ $a=-50 \mathrm{mV}$.
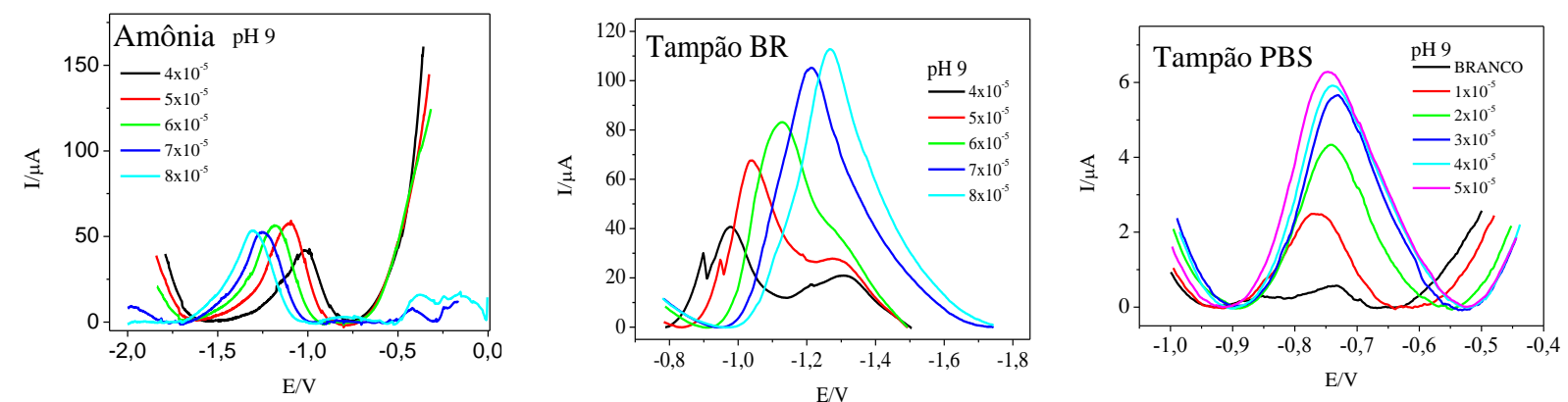

Figura 2: Voltamogramas de onda quadrada para a redução de $3,0 \times 10^{-5} \mathrm{M}$ de Bisfenol A, sobre AgSAE em tampão PBS, com $f=100 \mathrm{~s}^{-1}, \Delta \mathrm{E}_{\mathrm{s}}=-2 \mathrm{mV}$ e $a=-50 \mathrm{mV}$ e diferentes valores de $\mathrm{pH}$.

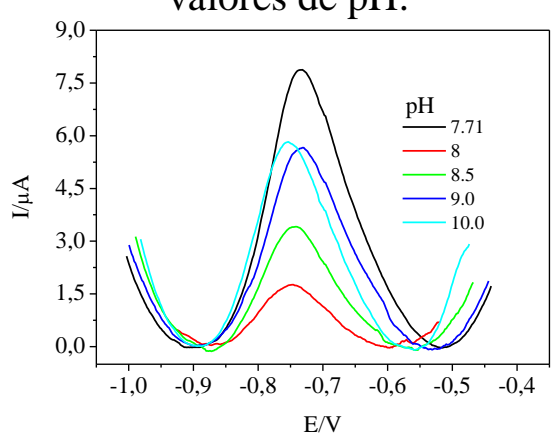

A fim de aumentar a sensibilidade e a seletividade do método, variou-se alguns parâmetros. Primeiramente testou a influência de surfactantes (catiônico, neutro e aniônico), com o uso de brometo de cetil trimetil amônio (CTAB), Triton X-100 (Triton) e lauril sulfato 


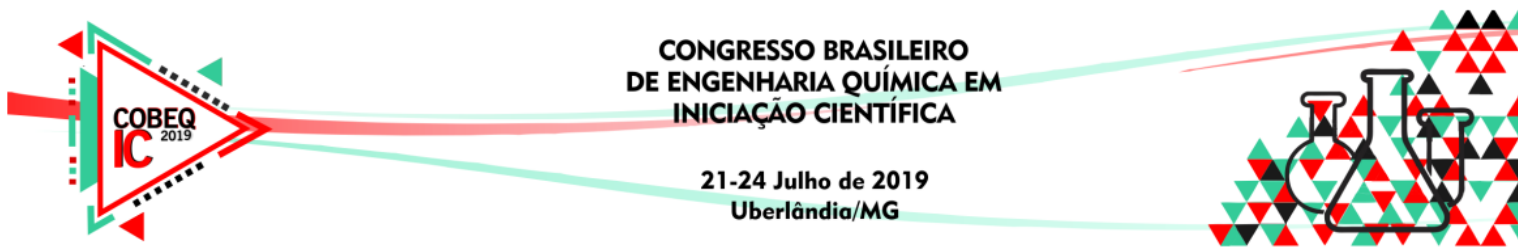

de sódio (SDS), respectivamente. Foram avaliados, porém não obteve melhor repetibilidade, reprodutibilidade e intensidade de sinal analítico, como é mostrado na Figura 5.

Figura 5: Voltamogramas de onda quadrada para a redução de 3,0 $\times 10^{-5} \mathrm{M}$ de Bisfenol A sobre AgSAE em tampão PBS em pH 7,71 com $f=100 \mathrm{~s}^{-1}, \Delta \mathrm{E}_{\mathrm{s}}=-2 \mathrm{mV}$ e $a=-50 \mathrm{mV}$, empregando-se diferentes surfactantes.
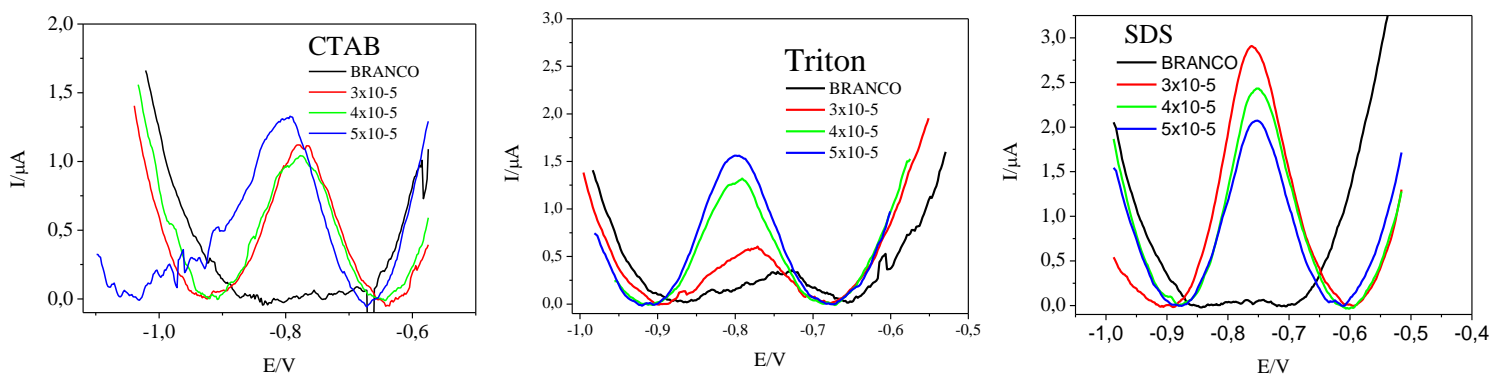

Posteriormente avaliou-se os parâmetros da SWV para obter um método mais sensível e seletivo. Primeiramente, foi avaliado o incremento de varredura de potencial de $-2 \mathrm{mV}$ a - $10 \mathrm{mV}$, observando que o sinal analítico aumentou proporcionalmente com o aumento do incremento, porém o pico voltametrico se deslocou para valores mais negativos, acompanhados de maior largura do pico, indicando perda da seletividade analítica, como pode ser observado na Figura 6. A amplitude dos pulsos de potencial foi avaliada de $-5 \mathrm{mV}$ a $-50 \mathrm{mV}$, onde foi observado que o aumento de $a$ promoveu aumento proporcional nos valores de corrente sem alteração nos potenciais de pico e na largura dos voltamogramas, como mostrado na Figura 6. A frequência de aplicação dos pulsos de potencial foi variada de $10 \mathrm{~s}^{-1} \mathrm{a}$ $300 \mathrm{~s}^{-1}$, observando que o sinal da corrente aumentou com o aumento no valor de $f$, conforme observado na Figura 6.

Figura 6: Voltamogramas de onda quadrada para a redução de $3,0 \times 10^{-5} \mathrm{M}$ de Bisfenol A sobre $\operatorname{AgSAE}, \operatorname{com} f=100 \mathrm{~s}^{-1}, a=5 \mathrm{mV}$ e $\Delta \mathrm{E}_{\mathrm{s}}=-2 \mathrm{mV}$. Variação individual de cada um dos parâmetros da VOQ.
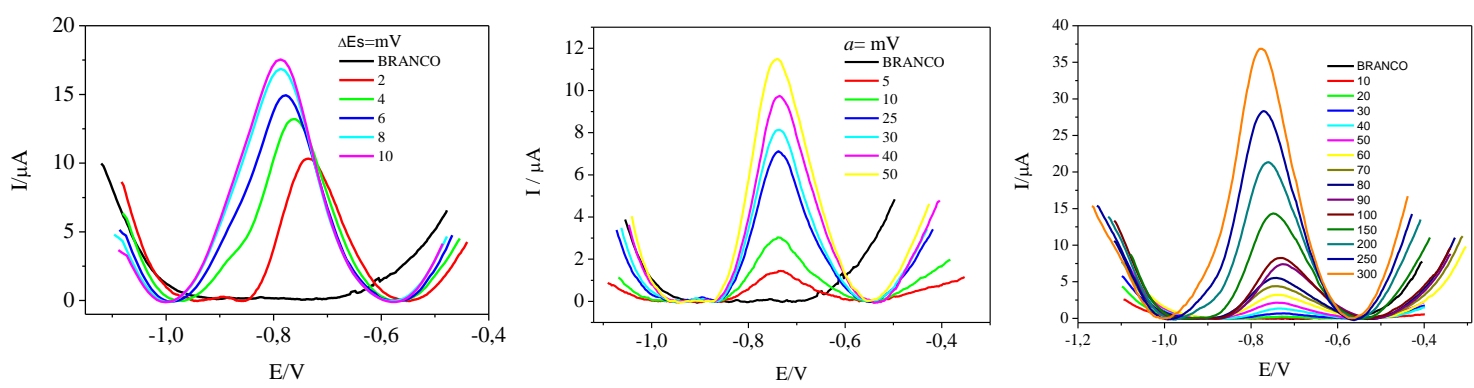

A avaliação de todos os parâmetros voltamétricos para se empregar uma metodologia mais sensível e seletiva mostrou que com amplitude de $-50 \mathrm{mV}$, incremento de varredura de potenciais de $-10 \mathrm{mV}$ e frequência de pulsos de potencial de $300 \mathrm{~s}^{-1}$, são adequados, porém ao construir as curvas analíticas empregando-se estes parâmetros observou-se um aumento 


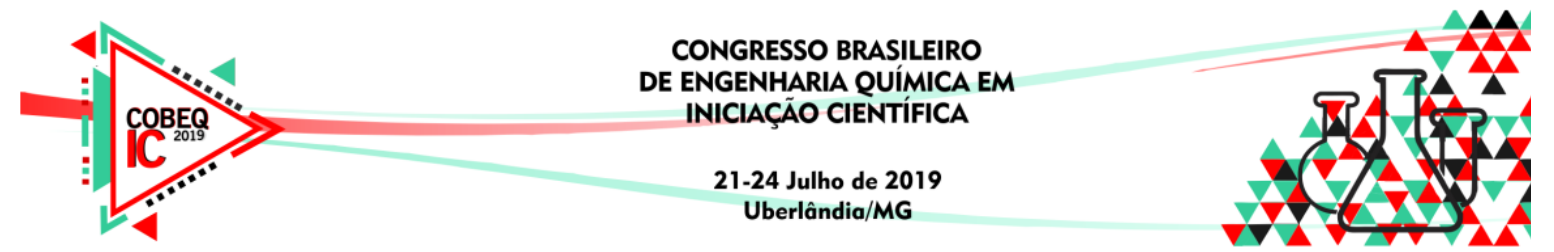

exacerbado de ruídos de fundo, os quais prejudicaram a metodologia proposta. Assim, novas curvas analíticas foram construídas etilizando-se amplitude de $-50 \mathrm{mV}$, incremento de $-2 \mathrm{mV}$ e a frequência de $100 \mathrm{~s}^{-1}$. Desta maneira, curvas de calibração foram construídas empregando-

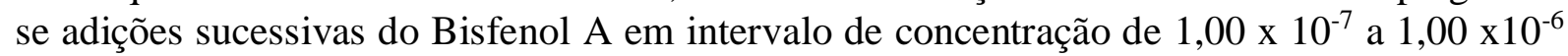
mol L ${ }^{-1}$ em uma solução de tampão PBS pH 7,71, onde observou-se aumento linear das correntes de pico com o aumento da concentração de Bisfenol A, indicando que o método proposto é sensível e poderá ser empregado com sucesso para a detecção em amostras de alimentos.

\section{CONCLUSÃO}

Devido a gravidade do Bisfenol A à saúde da população é de extrema importância aplicar esta metodologia e avaliar os possíveis interferentes em amostras complexas, como alimentos industrializados aquecidos e armazenados em embalagens plásticas contendo Bisfenol A. A metodologia proposta apresentou sensibilidade adequada, com boa seletividade, reprodutibilidade e repetibilidade, minizando-se assim os custos nas análises e o tempo necessário para a obtenção da informação analítica.

\section{REFERÊNCIAS}

DE SOUZA, D. et al. Voltametria de onda quadrada. Segunda parte: aplicações. Química nova, v. 27, n. 5, p. 790-797, out./jan. 2004.

DE SOUZA, D., et al. Utilização de eletrodos sólidos de amálgama para a determinação analítica de compostos orgânicos e inorgânicos. Química Nova, v. 34, n.03. p.487-496, 2011.

RAGAVAN, K. V et al. Sensors and biosensors for analysis of bisphenol-A. Trends in Analytical Chemistry, v.52, p.248-260, 2013.

SENCI, R. Efeitos do bisfenol A: um desregulador endócrino. Assis, 2015. Monografia (Bacharel em Química). Instituto Municipal de Ensino Superior de Assis, Fundação Educacional do Município de Assis.

STOFFERS, N. Certified reference materials for food packaging specific migration tests: development, validation and modelling. Wageningen, 2004. Monografia (Ph.D., embalagens - interação alimentar). Universidade de Wageningen.

WANG, Joseph. Analytical Electrochemistry. 3. ed. New Jersey: Wiley-vch, 2006. 250 p. 